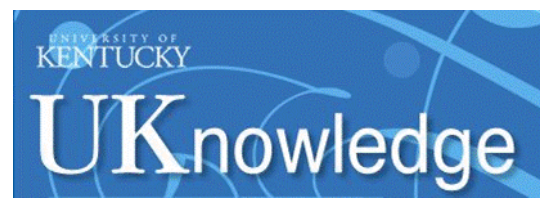

University of Kentucky

UKnowledge

8-16-2013

\title{
Controlled Magnetic Reversal in Permalloy Films Patterned into Artificial Quasicrystals
}

Vinayak Shantaram Bhat

University of Kentucky, vinayak8582@gmail.com

\section{J. Sklenar}

Northwestern University

B. Farmer

University of Kentucky

J. Woods

University of Kentucky

Jeffrey Todd Hastings

University of Kentucky, todd.hastings@uky.edu

See next page for additional authors

Follow this and additional works at: https://uknowledge.uky.edu/physastron_facpub

Part of the Electrical and Computer Engineering Commons, and the Physics Commons

Right click to open a feedback form in a new tab to let us know how this document benefits you.

\section{Repository Citation}

Bhat, Vinayak Shantaram; Sklenar, J.; Farmer, B.; Woods, J.; Hastings, Jeffrey Todd; Lee, S. J.; Ketterson, J. B.; and De Long, Lance E., "Controlled Magnetic Reversal in Permalloy Films Patterned into Artificial Quasicrystals" (2013). Physics and Astronomy Faculty Publications. 4.

https://uknowledge.uky.edu/physastron_facpub/4

This Article is brought to you for free and open access by the Physics and Astronomy at UKnowledge. It has been accepted for inclusion in Physics and Astronomy Faculty Publications by an authorized administrator of UKnowledge. For more information, please contact UKnowledge@lsv.uky.edu. 


\section{Controlled Magnetic Reversal in Permalloy Films Patterned into Artificial Quasicrystals}

Digital Object Identifier (DOI)

http://dx.doi.org/10.1103/PhysRevLett.111.077201

Notes/Citation Information

Published in Physical Review Letters, v. 111, no. 7, 077201.

(C) 2013 American Physical Society.

The copyright holder has granted the permission for posting the article here.

Authors

Vinayak Shantaram Bhat, J. Sklenar, B. Farmer, J. Woods, Jeffrey Todd Hastings, S. J. Lee, J. B. Ketterson, and Lance E. De Long 


\title{
Controlled Magnetic Reversal in Permalloy Films Patterned into Artificial Quasicrystals
}

\author{
V. S. Bhat, ${ }^{1}$ J. Sklenar, ${ }^{2}$ B. Farmer, ${ }^{1}$ J. Woods,${ }^{1}$ J. T. Hastings, ${ }^{3}$ S. J. Lee, ${ }^{2}$ J. B. Ketterson, ${ }^{2}$ and L. E. De Long ${ }^{1, *}$ \\ ${ }^{1}$ Department of Physics and Astronomy, University of Kentucky, 505 Rose Street, Lexington, Kentucky 40506, USA \\ ${ }^{2}$ Department of Physics and Astronomy, Northwestern University, 2145 Sheridan Road, Evanston, Illinois 60201, USA \\ ${ }^{3}$ Department of Electric and Computer Engineering, University of Kentucky, Lexington, Kentucky 40506, USA
}

(Received 19 May 2013; revised manuscript received 12 July 2013; published 12 August 2013)

\begin{abstract}
We have patterned novel Permalloy thin films with quasicrystalline Penrose $P 2$ tilings and measured their dc magnetization and ferromagnetic resonance absorption. Reproducible anomalies in the hysteretic, low-field data signal a series of abrupt transitions between ordered magnetization textures, culminating in a smooth evolution into a saturated state. Micromagnetic simulations compare well to experimental $\mathrm{dc}$ hysteresis loops and ferromagnetic resonance spectra and indicate that systematic control of magnetic reversal and domain wall motion can be achieved via tiling design, offering a new paradigm of magnonic quasicrystals.
\end{abstract}

Ferromagnetic (FM) thin films patterned with antidot lattices (ADLs) are candidates for ultra-high-density data storage [1]. Periodic FM ADLs are analogous to photonic crystals [2], exhibit energy band structure, and can be viewed as magnetic metamaterials [3]. Related wire network patterns have also attracted attention as examples of artificial spin ice [4-6].

The vast majority of previous ADL studies have been done on simple dot or antidot (AD) shapes (circle, square, etc.) patterned on periodic lattices (square, kagome, etc.) [7]. Simulations of periodic ADLs exhibit a reproducible evolution of FM domain walls (DWs) in the low-field, reversal regime critical to magnetic switching applications [8]. This high degree of reproducibility contrasts with the uncontrolled evolution of disordered DW textures under applied magnetic fields in unpatterned FM films and bulk solids.

Artificial quasiperiodic ADLs in FM thin films offer an interesting alternative to periodic ADLs but have been largely ignored. Quasiperiodic "tilings" are generated by explicit rules that dictate long-range order without periodic translational symmetry [9-11]; yet they are locally selfsimilar, and any finite-area pattern occurs infinitely many times within an arbitrarily large tiling. "Infinite" Penrose tilings possess tenfold rotational symmetry that is inconsistent with periodic lattices.

In this Letter, we report dc magnetization data and ferromagnetic resonance (FMR) spectra for ADLs formed by quasiperiodic Penrose $P 2$ tilings (P2Ts) [11]. Effects of relaxation of periodic symmetry on the control of magnetic reversal, DW motion, and therefore, spin wave propagation and dispersion in the hysteretic regime are our primary interests.

We have numerically generated P2Ts from "kites" and "darts" using a deflation method [9-11] that we incorporated into our electron beam lithography software. The nonmagnetic interior ( $\mathrm{Si}$ substrate) of each kite or dart forms an $\mathrm{AD}$ whose Permalloy boundaries have a width $W$, as shown in Fig. 1, where the reduced fivefold rotational symmetry without translational symmetry of the finite P2T is apparent. P2T can be viewed as a continuous wire network of two segment types with long or short lengths $d_{1}$ and $d_{2}$, respectively, and common width $W$. Sample films were deposited on $\mathrm{Si}$ substrates using electron beam evaporation, followed by lift-off. All P2Ts discussed herein have Permalloy film thickness $t=25 \mathrm{~nm}$. All sample parameters are listed in Table I.

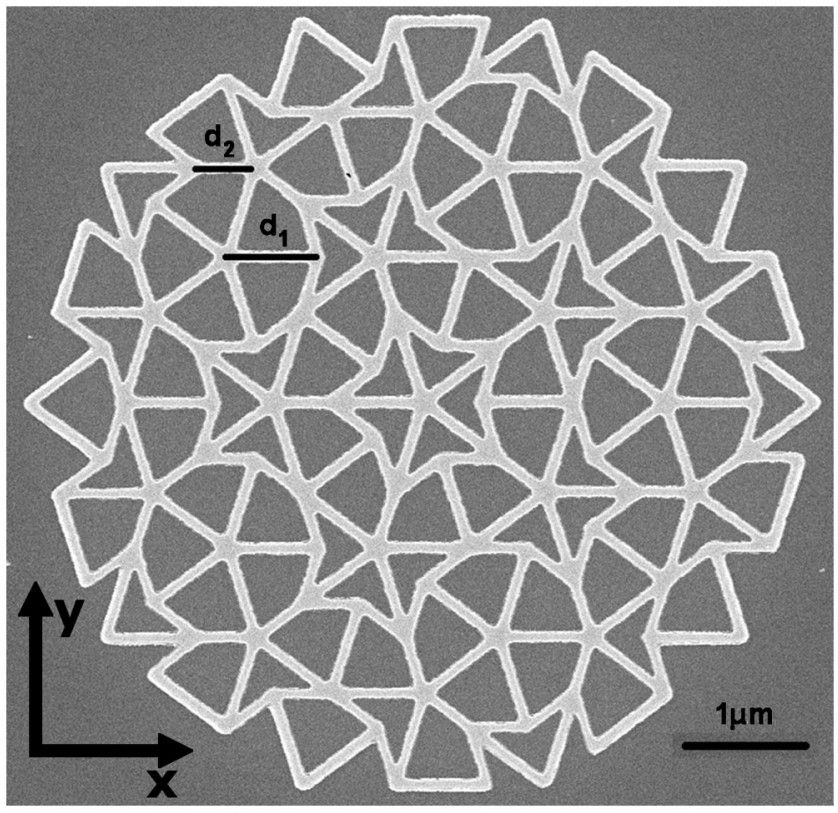

FIG. 1. SEM image of third-generation pattern of sample III134E. Bright (dark) regions correspond to permalloy ( $\mathrm{Si}$ substrate). Segment lengths are $d_{1}=810 \mathrm{~nm}$ and $d_{2}=500 \mathrm{~nm}$, and the width $W=85 \mathrm{~nm}$. An applied dc field $\boldsymbol{H}$ makes polar angle $\phi$ with respect to the $\hat{x}$ axis shown (defined as $\phi=0^{\circ}$ ) parallel to a decahedral base. 
TABLE I. Parameters of P2 tilings.

\begin{tabular}{lccr}
\hline \hline Sample & Generation & $d_{1}, d_{2}(\mathrm{~nm})$ & $W(\mathrm{~nm})$ \\
\hline III129A & 8 & 810,500 & 135 \\
III130C & 8 & 810,500 & 90 \\
III133C & 12 & 1618,1000 & 107 \\
III133E & 3 & 8100,5000 & 1100 \\
III134E & 3 & 810,500 & 85 \\
simulation & 3 & 810,500 & 100 \\
\hline \hline
\end{tabular}

Broadband FMR measurements were performed at microwave frequencies $10 \mathrm{MHz} \leq f \leq 20 \mathrm{GHz}$ and dc applied magnetic fields $-8 \mathrm{kOe} \leq H \leq+8 \mathrm{kOe}$, using a meander line method [12]. In all FMR experiments, the dc field could be rotated within the film plane by angle $\phi$ between $\boldsymbol{H}$ and a reference edge of the ADL ( $\hat{x}$ axis in Fig. 1). Static magnetization measurements were performed using a Quantum Design MPMS SQUID Magnetometer. Static magnetization and dynamic FMR data were simulated using the Object Oriented Micromagnetic Framework (OOMMF) code [13] and are limited to a third-generation P2T at $T=0 \mathrm{~K}$ (see Table I).

More detailed descriptions of our experimental and numerical techniques are given in the Supplemental Material [14].

Experimental DC magnetizations $M(H, T)$ for three P2T samples are shown in Fig. 2(a), and hysteresis loops for third-generation sample III134E at temperatures $T=$ $310 \mathrm{~K}$ and $5 \mathrm{~K}$ are shown in Fig. 2(b). The $M(H)$ data for third-generation sample III133E $(W=1.1 \mu \mathrm{m})$ exhibit a much weaker coercivity than those for thirdgeneration sample III134E, which has a much smaller value of $W=85 \mathrm{~nm}$ (note the exchange length (DW width) $\approx 6 \mathrm{~nm}$ for permalloy [15]). Given an expected increase of coercive field $H_{C}$ with decreasing $T$, the $M(H, T)$ data are in good agreement with a micromagnetic simulation performed for $T=0 \mathrm{~K}$ [black curve in Fig. 2(b)].

Figure 2 reveals pronounced knee anomalies in all experimental $M(H, T)$ data for 3rd, 8th, and 12th generation P2Ts acquired at $T>0$. Smaller, more numerous knees are visible in simulated $(T=0)$ hysteresis loops for three P2Ts with $W=50,100$, and $250 \mathrm{~nm}$ (Supplemental Material [14]). $M(H, T)$ data for sample III134E exhibit a prominent knee at $H \approx-0.20 \mathrm{kOe}$ for $T=5 \mathrm{~K}$, which compares well with the first large knee seen at $H \approx$ $-0.33 \mathrm{kOe}$ in a simulation of a P2T with the same segment aspect ratio $\left(d_{1} / W\right)$ and generation [see Fig. 2(b)]. Both experimental and simulated knees in $M(H, T)$ reproduce in opposite field sweeps; however, Fig. 2 shows that the size and number of the knees depend on generation (tiling area) and temperature.

Simulated dc magnetization maps show the knees can be associated with abrupt reversals of subsets of P2T segments (Supplemental Material [14]). A map
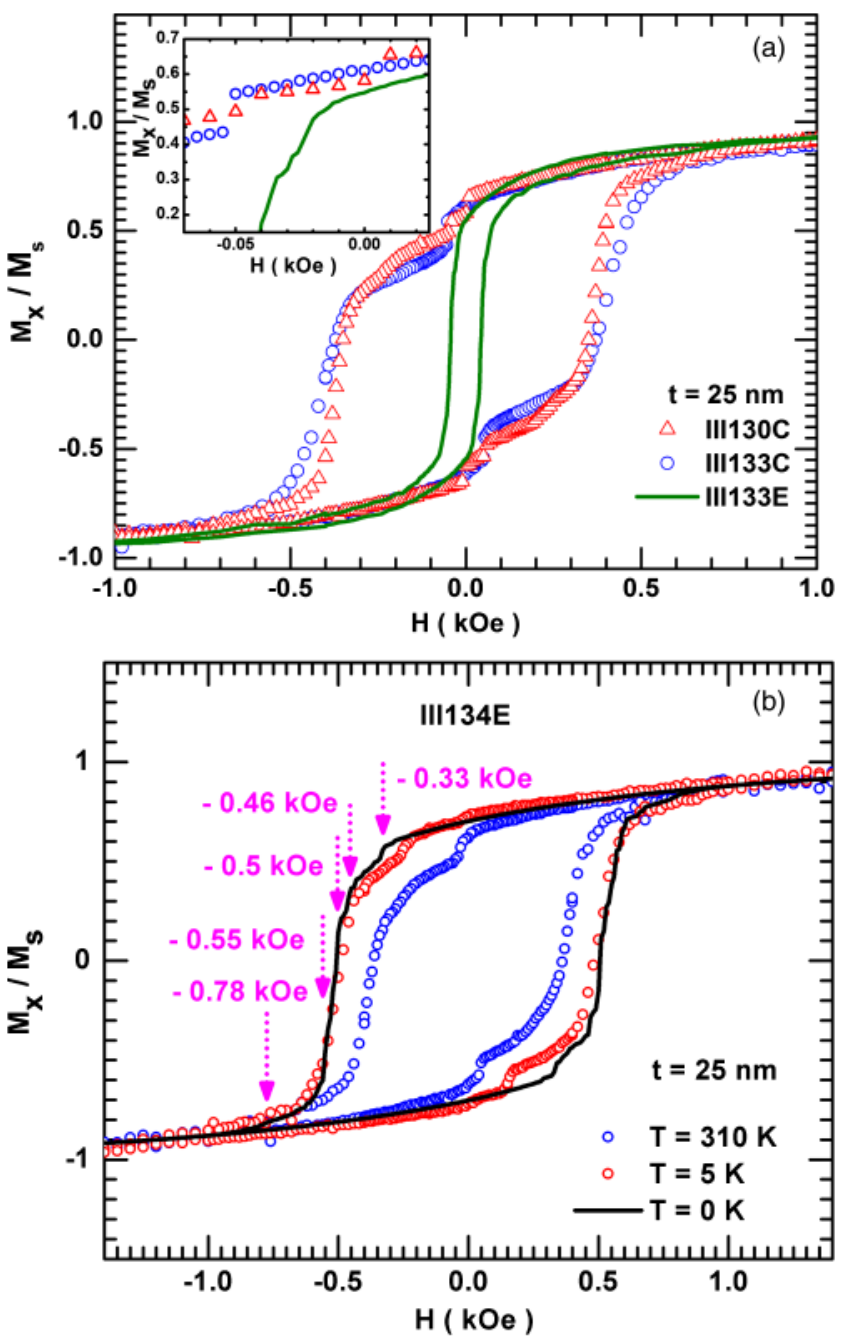

FIG. 2 (color online). (a) Horizontal dc magnetization $M_{X}$ normalized to saturation value $M_{S}$ vs applied dc magnetic field $H$ for samples III130C (8th gen.), III133C (12th gen.), and III133E (3rd gen.) at temperature $T=310 \mathrm{~K}$. Inset shows first knees encountered in a negative field sweep. (b) $M_{X} / M_{S}$ for sample III134E vs $H$ for $T=310$ and $5 \mathrm{~K}$, compared with the simulated magnetization (black line) at $T=0 \mathrm{~K}$. Knees in simulated $M(H)$ are indicated by arrows.

for a third-generation P2T of $W=100 \mathrm{~nm}$ at $\boldsymbol{H}=$ $(-0.46 \mathrm{kOe}) \hat{x}$ during a "negative field sweep" (defined herein as $+12 \mathrm{kOe}$ to $-12 \mathrm{kOe}$ ) is shown in Fig. 3(a), where five distinct subsets of film segments oriented at angles of $0^{\circ}, 72^{\circ}, 144^{\circ}, 216^{\circ}$, and $288^{\circ}$ with respect to $\boldsymbol{H}$ are apparent. The near-uniform segment polarizations parallel to their axes evident in Fig. 3(a) persist over a range of applied fields. Consequently, any vertex (formed by joining three to five segments in the P2T) must confine DWs, and abrupt switching events of entire segments are expected with changing field.

It is instructive to relate experimental knee events to sharp, reproducible anomalies in the FMR absorption derivative. Spectra for sample III129A at frequency 

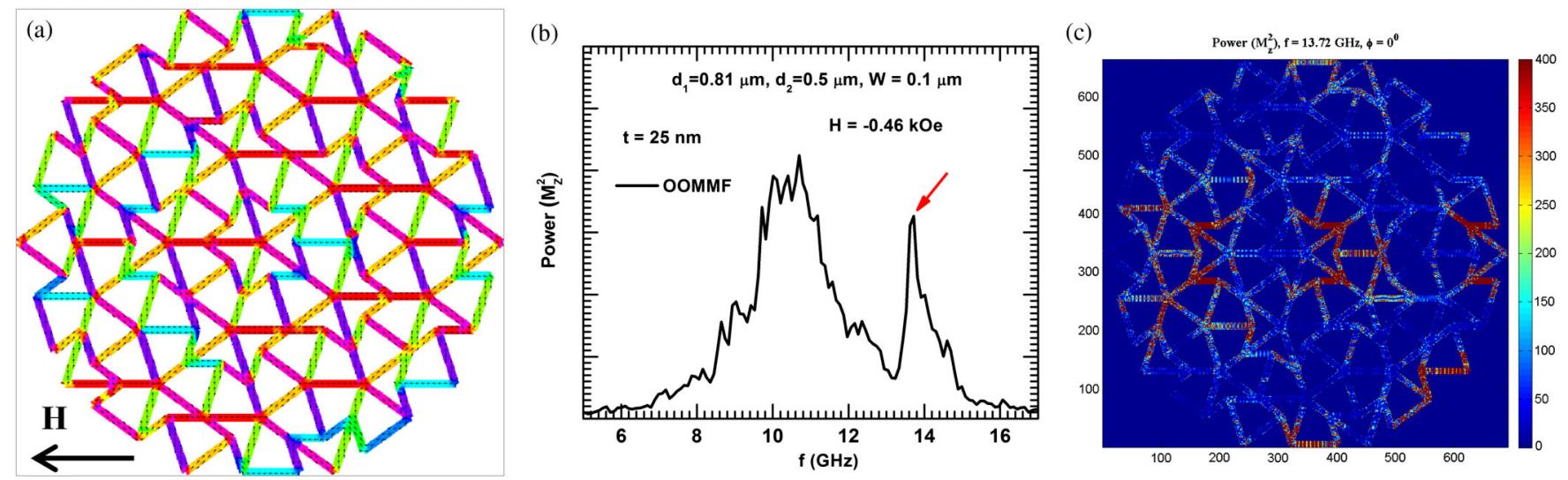

FIG. 3 (color online). (a) Simulated dc magnetization map for $H=-0.46 \mathrm{kOe}$. The arrow denotes dc field direction, and the color scale indicates individual pixel polarization. Arrows indicate average direction of $\approx 10^{2}$ pixels. Horizontal $\left(0^{\circ}\right.$, red) segments begin switching (to $180^{\circ}$, turquoise) at $H=-0.29 \mathrm{kOe}$ [14]. (b) Simulated absorbed power (squared spin tipping amplitude) vs frequency $f$ for $H=-0.46 \mathrm{kOe}$. A red arrow marks a peak (that first emerges for $H \approx-0.3 \mathrm{kOe}$ and $f \approx 13.4 \mathrm{GHz}$ ) that corresponds to the appearance of the first large knee in $M(H)$ in Fig. 2(b) and the first flipped $0^{\circ}$ segment [14]. (c) Simulated power absorption map for $H=-0.46 \mathrm{kOe}$ and $f=13.72 \mathrm{GHz}$, corresponding to (a) and (b). Color scale indicates power amplitude (red is greatest). Note the correlation between locations of switched (turquoise) segments in (a) and resonant (red) segments with several orientations.

$f=13 \mathrm{GHz}$ are shown in Fig. 4(a) and were acquired in fields $\boldsymbol{H}=H \hat{x}$ that span the lower-field, strongly hysteretic (e.g., $|H|<1 \mathrm{kOe})$ and near-saturated $(|H|>1 \mathrm{kOe})$ regimes. All resonance signatures quantitatively reproduce in opposite field sweeps. The remarkable reproducibility of the mode signatures throughout the field sweeps suggests that pinning of the magnetization parallel to the AD edges and confinement of DWs at P2T vertices controls segment polarization and low-field reversal.

We observe two distinctly different types of modes depending on the dc field strength: in the near-saturated regime, "symmetric" mode signatures repeat on both sides of the field origin, despite finite hysteresis. "Asymmetric" modes, defined by their presence on only one side of the field origin in a given sweep, are found only in the lowerfield regime, as are knees in $M(H, T)$. Experimental frequency-field mode dispersion curves for sample III129A are shown in Fig. 5, including two asymmetric mode branches 10 and 11 (Supplemental Material [14]): one mode lies at $H=-50 \mathrm{Oe}(+50 \mathrm{Oe})$ for $f=13 \mathrm{GHz}$ for negative (positive) field sweeps [Fig. 4(a)]. Our simulations for $\mathrm{P} 2 \mathrm{~T}$ with $W=100 \mathrm{~nm}$ show that modes in the (a)

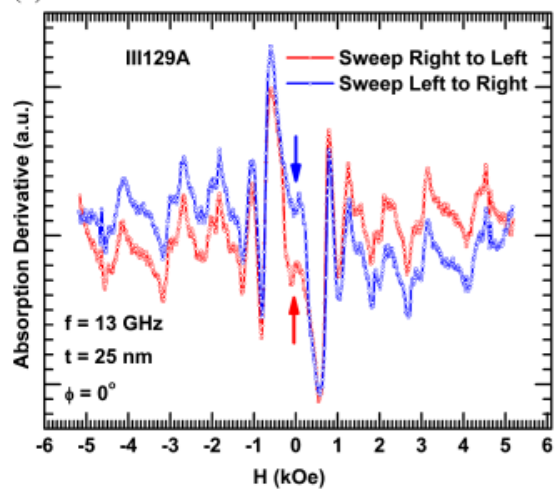

(b)



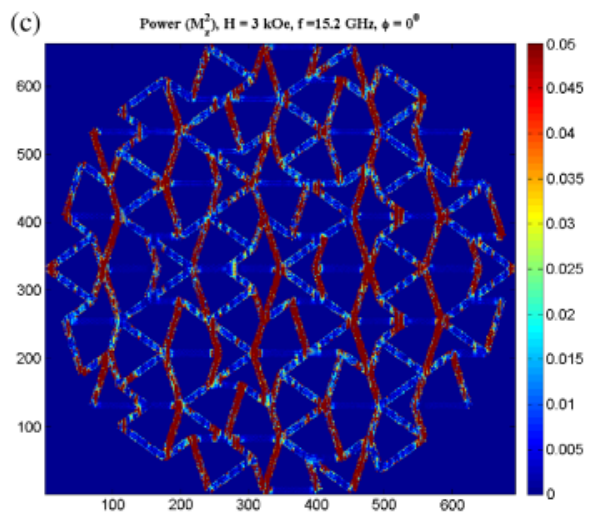

FIG. 4 (color online). (a) Experimental FMR absorption of sample III129A for frequency $f=13 \mathrm{GHz}$ and temperature $T=310 \mathrm{~K}$. Note reproducible, symmetric spectra for dc fields $|H|>1 \mathrm{kOe}$; arrows mark an asymmetric mode that only occurs on one side of the field origin near $|H|=50$ Oe. (b) Simulated global power vs frequency $f$ in applied field $\boldsymbol{H}=(3 \mathrm{kOe}) \hat{x}$ and $T=0 \mathrm{~K}$ for a thirdgeneration P2T with segment dimensions shown. Data in (a) suggest that the modes for $H=3 \mathrm{kOe}$ are symmetric. (c) Simulated power absorption map for $f=15.2 \mathrm{GHz}$ and $\boldsymbol{H}=(3.0 \mathrm{kOe}) \hat{x}$, corresponding to a symmetric mode peak [red arrow in (b)]. The color scale indicates that magnetization tipping amplitude (red is greatest) is concentrated in extended chains of segments oriented at $\phi= \pm 72^{\circ}$ and $\pm 108^{\circ}$. 


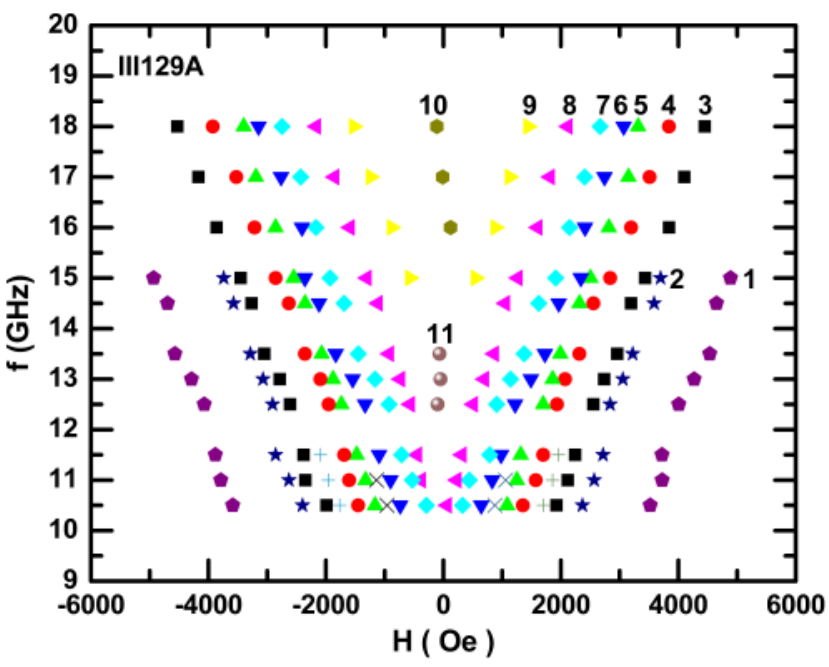

FIG. 5 (color online). FMR frequency $f$ vs dc field $H$ for sample III129A. Numbers denote mode branches; 10 and 11 correspond to asymmetric modes such as those shown in Fig. 3(c). Note that two additional branches (not numbered) appear below $f=12 \mathrm{GHz}$. Associations of branches with particular dc magnetization and power maps are detailed in the Supplemental Material [14].

near-saturated regime are better dispersed, consistent with Fig. 5, and are strongly correlated with subsets of segments oriented at particular angles with respect to $\boldsymbol{H}$ (Supplemental Material [14]), as suggested by Fig. 4(c).

On the other hand, dc magnetization and power maps for the reversal regime show that the first knees in $M(H)$ mark the switching of segment polarizations and the appearance of FMR power absorption within and near the switched horizontal segments; e.g., the first knee at $H=-0.29 \mathrm{kOe}$ marks the first abrupt reversal of a horizontal segment (Supplemental Material [14]). Additional horizontal segments switch [red to turquoise in Fig. 3(a)] as $H$ is decreased over the interval $-0.46<H<-0.29 \mathrm{kOe}$; simultaneously, a strong FMR peak grows and disperses after emerging at $f=13.4 \mathrm{GHz}$ for $H=-0.29 \mathrm{kOe}$ [Fig. 3(b)]. The power absorption map in Fig. 3(c) shows that the low-field modes are not restricted to particular subsets of segments having one common orientation (e.g., $\phi=0^{\circ}$ or $180^{\circ}$ ) with respect to the dc field.

Remarkably, our FMR spectra for sample III129A exhibit tenfold rotational symmetry for $|H|>1 \mathrm{kOe}$, as shown in Fig. 6, despite the fivefold symmetry of our finite P2T sample. FMR spectra for sample III133E also exhibit tenfold symmetry over the entire near-saturated interval $(0.78<H<1.2 \mathrm{kOe})$ where modes were detected (Supplemental Material [14]). Tenfold rotational symmetry could indicate that the demagnetizing fields of segments on the edges of third-generation decagons are too weak to break the tenfold symmetry expected for an infinite P2T. Alternatively, a rotation of our finite P2T by $180^{\circ}$ causes segments at field angles $\phi= \pm 36^{\circ}\left( \pm 72^{\circ}\right)$

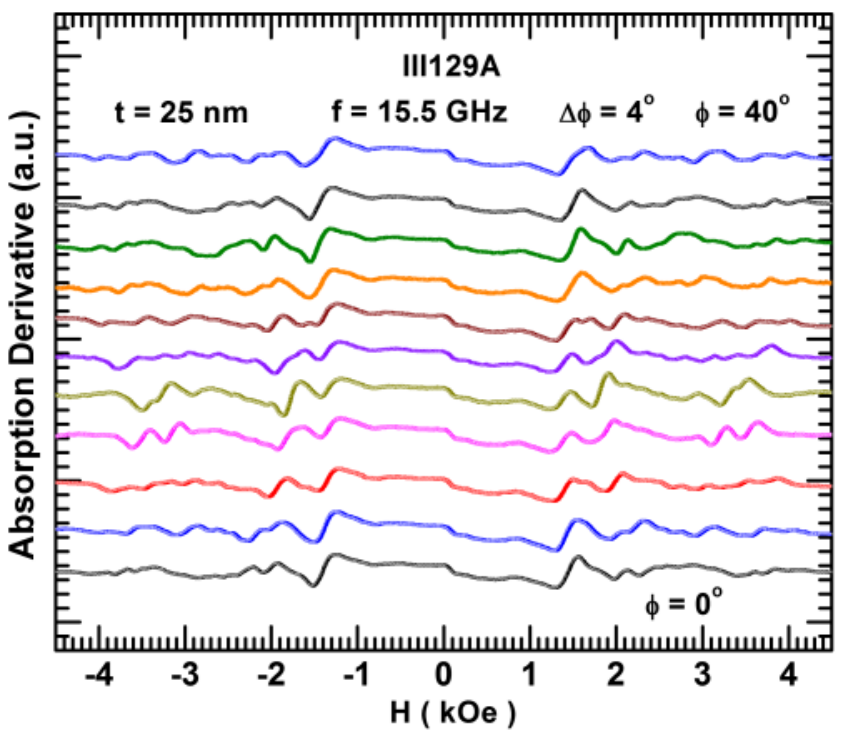

FIG. 6 (color online). FMR absorption derivative vs applied dc magnetic field $H$ of sample III129A in negative field sweeps at $T=300 \mathrm{~K}$ for frequency $f=15.5 \mathrm{GHz}$. Colors correspond to different angles $0^{\circ} \leq \phi \leq 40^{\circ}$ between the decagon base and $\boldsymbol{H}$ (see Fig. 1). Adjacent curves correspond to angular increments $\Delta \phi=4^{\circ}$. Note tenfold rotational symmetry (black and blue curves reproduce for $\Delta \phi=36^{\circ}$ ).

to make angles of $\pm 144^{\circ}\left( \pm 108^{\circ}\right)$, respectively; however, the number of segments with uniform polarizations oriented at particular angles with respect to $\boldsymbol{H}$ remains unchanged. If field-segment orientation is the main factor controlling FMR response in a near-saturated regime, the total power absorption will exhibit tenfold symmetry at higher fields.

In summary: (1) We have fabricated a novel class of FM films patterned into quasiperiodic P2Ts that exhibit longrange order, but no periodic translational symmetry. (2) Reproducible knees in the dc magnetization occur in the low-field regime. The number and size of the knees depends upon temperature and P2T generation (sample area), suggesting that cooperative behavior exists among segments. (3) Simulations in the low-field regime reveal that switching of individual segments is accompanied by local FMR absorption among clusters of segments having several orientations with respect to dc field. (4) The orientation of film segments with respect to dc field strongly influences static and dynamic magnetization in the nearsaturated regime. (5) FMR spectra for finite P2Ts exhibit tenfold rotational symmetry in the near-saturated regime, which is only expected for infinite P2Ts.

Our results show that aperiodic patterning provides a powerful method for controlling magnetic reversal and dynamics in FM thin films. The observed pinning of the magnetization by segment edges and confinement of DWs at vertices in quasiperiodic lattices should strongly affect spin wave propagation, which implies that P2Ts constitute a new paradigm of magnonic quasicrystals. 
Research at the University of Kentucky was supported by U.S. DOE Grant No. DE-FG02-97ER45653 and the UK Center for Advanced Materials (supported by U.S. NSF Grant No. EPS-0814194), and the UK Center for Computational Sciences. We thank Professor David Rabson for critical discussions.

*Corresponding author. delong@pa.uky.edu

[1] R. Cowburn, A. Adeyeye, and J. Bland, Appl. Phys. Lett. 70, 2309 (1997).

[2] E. Yablonovitch, Phys. Rev. Lett. 58, 2059 (1987).

[3] S.O. Demokritov and A.N. Slavin, Magnonics: From Fundamentals to Applications (Springer, New York, 2013), Vol. 125.

[4] R. F. Wang et al., Nature (London) 439, 303 (2006).

[5] W. Branford, S. Ladak, D. E. Read, K. Zeissler, and L. F. Cohen, Science 335, 1597 (2012).

[6] S. Gliga, A. Kakay, R. Hertel, and O. G. Heinonen, Phys. Rev. Lett. 110, 117205 (2013).
[7] C. Yu, M. J. Pechan, and G. J. Mankey, Appl. Phys. Lett. 83, 3948 (2003).

[8] V. Bhat, J. Woods, L.E. De Long, J. T. Hastings, V. V. Metlushko, K. Rivkin, O. Heinonen, J. Sklenar, and J.B. Ketterson, Physica (Amsterdam) 479C, 83 (2012).

[9] A. Glassner, IEEE Comput. Graph. Appl. 18, 83 (1998).

[10] N. G. De Bruijn, Indagat. Math 43, 38 (1981).

[11] E.W. Weisstein, Penrose Tiles (http:// mathworld.wolfram.com/PenroseTiles.html).

[12] C.-C. Tsai, J. Choi, S. Cho, S. J. Lee, B. K. Sarma, C. Thompson, O. Chernyashevskyy, I. Nevirkovets, and J. B. Ketterson, Rev. Sci. Instrum. 80, 023904 (2009).

[13] M. Donahue and D. Porter, Interagency Report No. NISTIR 6376, 2002.

[14] See the Supplemental Material at http://link.aps.org/ supplemental/10.1103/PhysRevLett.111.077201 for technical details and additional experimental and numerical results.

[15] A. Guimaraes, Priniciples of Nanomagnetism (Springer, New York, 2009). 